\title{
Greco, Gallico ed Etrusco:Varrone e le componenti del Latino
}

1. Secondo una notizia varroniana riportataci da Giovanni Lido (de magistratibus, 2, 13), la lingua latina si comporrebbe di tre elementi costitutivi, il Greco (o meglio l'Eolico), il Gallico e l'Etrusco, in accordo alla teoria varroniana della

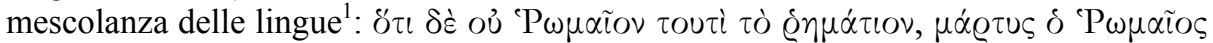

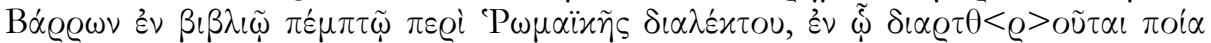

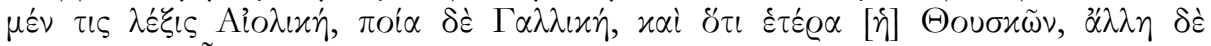

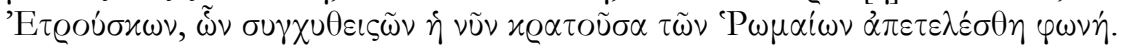

Il testo $^{2}$ di Giovanni Lido, fra gli altri dubbi che solleva, presenta un'immediata difficoltà esegetica: come spiegare il duplice e quindi pleonastico

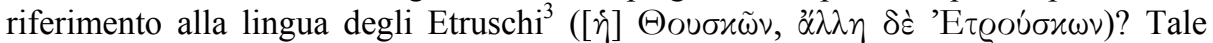
ridondanza è stata messa in relazione con un'altra apparente contraddizione del testo: la mancata menzione del Sabino. Come è possibile ${ }^{4}$, ci si è chiesti, che Varrone, che costantemente attribuiva un ruolo di primo piano ai Sabini come nella storia di Roma così nella formazione della lingua latina, non abbia citato in questo caso il Sabino

Desidero ringraziare il Professor Bruno Rochette (Université de Liège) per l'attenzione con cui ha seguito la stesura del presente lavoro. Mia ovviamente la responsabilità di quanto sostenuto nelle pagine seguenti.

1 Testo tratto da H. FunAIOLI, Grammaticae Romanae Fragmenta, Lipsiae, 1907, fr. 296 p. 311. Cf. anche l'edizione di G. Goetz, F. Schoell, M. Terenti Varronis de lingua Latina quae supersunt, Lipsiae, 1910, fr. 47 p. 201; A. Traglia, Marco Terenzio Varrone, Opere, Torino, 1974, fr. 8 p. 502.

2 Per il testo di Giovanni Lido rimando alla recente edizione di M. Dubuisson, J. Schamp, Jean le Lydien. Des magistratures de l'État romain, Paris, 2006, in particolare p. 18 e p. $149-150$

3 Pensano ad un errore della tradizione G. PASCUCCI, "Le componenti linguistiche del latino secondo la dottrina varroniana", in Scritti in onore di Benedetto Riposati, vol. 2, Rieti, 1979 , p. 339-363, in part. p. 339-342 (ivi bibliografia precedente) e F. CAVAZZA, Studio su Varrone etimologo e grammatico, Firenze, 1981, p. 88-97 e in part. p. 91-95 per la discussione relativa all'identificazione dell'opera varroniana da cui Giovanni Lido avrebbe tratto la citazione. Fondamentali le considerazioni, generali e specificamente relative a questo passo, di J. COllarT, Varron grammairien latin, Paris, 1954, p. 239. Ritiene invece che il testo abbia un suo preciso senso così come ci è stato tramandato D. BRIQUEL, "La conception du latin comme langue mixte chez Varron", in De lingua latina novae quaestiones. Actes $d u X^{e}$ colloque international de linguistique latine, Paris, 1999, p. 1033-1043. Secondo lo studioso, la duplice menzione di Etruschi e Tusci rifletterebbe la distinzione tra termini di origine etrusca (tramite i Tusci) più recente (e cioè gli abitanti dell'Etruria dei suoi tempi) e termini di origine più antica, tramite il riferimento agli Etrusci. Dubuisson, o.c. (n. 2), p. 150, sembra seguire Briquel. Si veda oltre per la discussione di questa ipotesi.

4 Sulla presunta aporia del testo di Giovanni Lido si è soffermato in particolare PASCUCCI, l.c. (n. 3), p. 340. 
come elemento costituente del Latino? E com'è possibile che invece sia citato il Gallico $^{5}$, il quale, stando proprio ad altre testimonianze varroniane, non ebbe che un minimo nonché episodico influsso sulla formazione del Latino?

Poiché dunque è apparsa insostenibile la mancata citazione del Sabino, è stato proposto di correggere il testo tràdito proprio nel punto della duplice e problematica citazione degli Etruschi, sostituendo ai Tusci gli Osci ${ }^{6}$. Con questa proposta si è pensato di risolvere entrambi i problemi rappresentati dal testo di Giovanni Lido: da una parte si è eliminato il secondo e pleonastico riferimento ai Tusci, dall'altra si è introdotto nel sistema "genetico" del Latino il Sabino, grazie alla menzione degli Osci. Poiché infatti, secondo Varrone, anche la lingua osca deriva da quella sabina (De lingua latina, VII, 3, 3), nel passo di Giovanni Lido il riferimento agli Osci rimanderebbe in realtà ai Sabini.

Di tutta la ricostruzione proposta a me pare particolarmente debole proprio il collegamento tra Osci e Sabini. Se infatti è vero che i Sabini, in Varrone, assurgono per così dire al ruolo di Italici in generale ${ }^{7}$, sarebbe del tutto sorprendente che Varrone, per citare i Sabini, si riferisse agli Osci, pur tenendo conto di un possibile legame di carattere mitico esistente tra i due popoli (tramite la mediazione sannitica, per cui De lingua latina, VII, 3, 5) e ricollegabile alla pratica del ver sacrum. Proprio a causa del ruolo di primo piano costantemente rivestito dai Sabini nella storia di Roma, sempre nell'ottica varroniana, ci si sarebbe aspettati una chiara ed esplicita citazione di questi, non un rimando criptico tramite gli Osci. Questa considerazione vale ancor di più se si considera che il discorso di Varrone si muove all'interno della discussione linguistica: non solo l'Osco è, per Varrone, una lingua ancora in uso (De lingua latina, $\mathrm{V}, 30$ ) distinta dal Latino, ma sembra anche che esso non abbia avuto un'influenza di grande importanza sul Latino, e quindi in nulla paragonabile al ruolo rivestito dal Sabino. Se quindi Varrone avesse fatto valere, anche solo nel caso del testo tramandatoci da Giovanni Lido, l'equazione Osco $=$ Sabino, sarebbe caduto in una contraddizione intollerabile per la sua ricostruzione etimologica del Latino e soprattutto per l'ideologia ad essa sottesa.

Se così è, e cioè se è impossibile introdurre, sulla base della tradizione, l'elemento sabino nella testimonianza di Giovanni Lido, come spiegarne l'assenza?

5 Secondo PASCUCCI, l.c. (n. 3), p. 352, "quanto agli elementi introdottisi nel lessico latino da altre lingue, e nella fattispecie dal celtico, essi in sostanza si limitano alla designazione di oggetti stranieri, il cui uso i Romani adottarono insieme al loro nome di origine". Questo è appunto il motivo per il quale Giovanni Lido, nella testimonianza in questione, cita il frammento varroniano. Su altri aspetti della tradizione varroniana in Giovanni Lido cf. Br. Rochette, "Jean le Lydien, Caton, Varron et Servius (Jean le Lydien, De magistratibus, I, 5)", ByzZ 91 (1998), p. 471-474.

6 Così PASCuCCI, l.c. (n. 3), p. 340 e nt. 4, secondo cui la somiglianza grafica tra

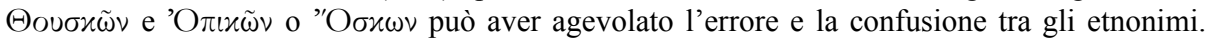
Precedentemente, pur non proponendo la medesima correzione sostenuta dal Pascucci, aveva espresso l'opinione che nel testo di Giovanni Lido vada inserito un riferimento all'elemento osco F. Della CoRTe, Varrone, il terzo gran lume romano, Firenze, 1979, p. 36. Italici”.

PASCUCCI, l.c. (n. 3), p. 345: "il termine Sabini sostituisce in pieno la nostra nozione di 
E' necessario prima di tutto soffermarsi su quanto affermato in positivo a proposito della mescolanza di lingue posta all'origine del Latino: come è stato giustamente messo in rilievo ${ }^{8}$, solo il Greco, tra le tre lingue menzionate, ebbe un ruolo importante nella formazione del Latino, anche nell'ottica varroniana. A questo proposito bisogna specificare che Varrone non era un sostenitore dell'origine "totale" del Latino dal Greco, non riteneva cioè che il Latino fosse interamente espressione del Greco, come pure alcuni grammatici antichi teorizzavano ${ }^{9}$. Al contrario, Varrone, pur attribuendo al Greco un ruolo di primaria importanza nella formazione del Latino (spesso grazie alla mediazione del Sabino), ritiene questa lingua "straniera" (peregrina) rispetto a quella latina (De lingua Latina, V, 20, 1), dunque fondamentalmente differente.

In secondo luogo dobbiamo prendere in considerazione un punto cruciale della teorizzazione etimologica varroniana, la commistione tra parole considerate "autoctone" (vernacula e domi nata) e parole considerate "straniere" (aliena o peregrina), o meglio ancora la distinzione tra i nostra e gli aliena verba: ad esempio, in De lingua latina, V, 1, 10 gli elementi costitutivi del Latino sono di tre tipi, nostra, aliena e oblivia; in De lingua Latina, V, 1, 12, a proposito dei nomi degli animali, si afferma che vocabula...partim sunt vernacula, partim peregrina. La precisazione (De lingua latina, $\mathrm{V}, 1,3)$ neque omnis origo est nostrae linguae e vernaculis verbis richiama l'esistenza, secondo Varrone, di una lingua originaria e autoctona (De lingua latina, $\mathrm{X}, 3,32)$, unum vernaculum ac domi natum, alterum adventicium, tertium nothum ex peregrino hic natum), su cui si sono innestati successivamente elementi linguistici stranieri di vario tipo.

In sintesi, Varrone sostiene la genuinità della lingua latina rispetto a quella greca $^{10}$, e soprattutto sostiene l'esistenza di un patrimonio lessicale autoctono, arricchito progressivamente dall'apporto di lingue percepite come straniere. Ci si è interrogati sull'identità di questo linguaggio vernacolare ${ }^{11}$, da considerare come vera e propria base del Latino, ma in questo senso Varrone non fornisce ulteriori indicazioni. Affermare infatti che il Latino è lingua autoctona non significa che Varrone non sostenesse l'esistenza di altre lingue italiche dotate della medesima caratteristica,

8 PASCUCCI, l.c. (n. 3), p. 341. Per l'apporto greco al Latino e soprattutto per il Latino inteso come dialetto greco, cf. E. GABBA, "Il latino come dialetto greco", in Studi alessandrini in memoria di A. Rostagni, Torino, 1963, p. 188-194. In sintesi, sembra potersi affermare che Varrone sostenesse l'origine eolica (intesa come componente della lingua) del Latino, determinata dalla presenza arcadica nel mito delle origini di Roma. Ciò non significa ovviamente che Varrone non valorizzasse anche la presenza nella lingua latina di elementi di origine diversa e non greca. F. Della CORTE, La filologia latina dalle origini a Varrone, Firenze, 1981, p. 166-172. Vd. anche Collart, o.c. (n. 3), p. 206-218 e FunAiOli, o.c. (n. 1), p. 443-448; BRIQUEL, l.c. (n. 3), p. 1035.

9 Su questo problema cf. in sintesi CAVAzZA, o.c. (n. 3), p. 89.

10 Ciò ovviamente non significa che Varrone sminuisse il ruolo del Greco (segnatamente l'eolico) nella costituzione del Latino, ma semmai che il Reatino accettasse la sinergia di più componenti linguistiche, di cui il Greco rappresentava verosimilmente la parte più "nobile". Cf. in particolare Della CORTE, o.c. (n. 8), p. 170.

11 CAvazZA, o.c. (n. 3), p. 96-97; COLLART, o.c. (n. 3), p. 249. 
soprattutto in considerazione del fatto che il Latino era percepito, anche da Varrone, come lingua mista e dunque non perfettamente autoctona (si è infatti parlato di "moderata autoctonia" del Latino ${ }^{12}$ ), escluso forse che per il suo livello più antico.

Questo particolare aspetto della teoria varroniana ci è noto grazie alla testimonianza di Dionigi di Alicarnasso, le cui nozioni linguistiche riposano "essentiellement sur ce qu'il a trouvé dans l'oeuvre du Réatin ${ }^{13}$ ". E' senz'altro vero che la necessità di dimostrare le origini greche di Roma fanno sì che Dionigi ${ }^{14}$ semplifichi in un'astratta dicotomia tra lingua greca e lingua barbara il complesso problema delle origini del Latino, così come si presentava in Varron $\mathrm{e}^{15}$. $\mathrm{E}$ ' però altrettanto vero che in questa suddivisione "grossolana" (almeno nella concezione dionigiana) è possibile ravvisare il ricordo di una più complessa ricostruzione linguistica, quale doveva essere esposta dal Reatino. In questa un ruolo centrale doveva essere certamente rivestito dalla teoria della lingua mixta, una lingua cioè alla cui formazione avevano concorso elementi di origine eterogenea. Di tale visione è esplicativo, a mio avviso, il passo in questione di Giovanni Lido, che, pur nella sua stringatezza e forse in un certo fraintendimento della fonte (almeno nella duplice e dunque pleonastica menzione della lingua degli Etruschi / Tusci, qualora non si accetti la soluzione prospettata da Briquel), rivela l'immagine varroniana della lingua latina.

Se sul Greco come componente della lingua molto è stato detto, non dobbiamo dimenticare il ruolo primario rivestito dalla lingua sabina nella formazione del Latino, non solo perché essa fornì a quest'ultima un gran numero di vocaboli, ma anche perché il Greco, come l'Etrusco, fu noto ${ }^{16}$ ai primi Romani, sempre secondo Varrone, grazie all'intermediazione sabina (Res rustica, III, 1).

Ha dunque ragione Briquel quando afferma che a differenza di altre lingue "indigene", il Sabino occupa una posizione di primo piano, secondo (quanto a numero di vocaboli) solo al Greco.

Questa constatazione conferma la problematicità del passo di Giovanni Lido e suggerisce la possibilità che l'assenza del Sabino non sia da attribuire ad un errore commesso da Giovanni Lido (o dalla sua fonte), ma corrisponda ad un preciso aspetto della teoria linguistica varroniana.

La questione, posta in questi termini, non può più risolversi nel tentativo di correggere, in modo forzoso, il passo di Giovanni Lido, in modo da farvi comparire anche il Sabino; semmai, sarà da verificare se Varrone abbia avuto un suo specifico motivo per non collocare il Sabino sullo stesso piano di Etrusco, Gallico e Greco.

2. A questo punto, per tentare di fornire una risposta al problema appena delineato, è forse opportuno saldare la ricostruzione storica delle fasi più antiche

12 Così CAVAZZA, o.c. (n. 3), p. 97

13 Briquel, l.c. (n. 3), p. 1037.

14 Per l'indicazione dei passi in cui Dionigi dipende, più o meno esplicitamene, da Varrone, cf. BRIQUEL, l.c. (n. 3), p. 1035-1038.

15 Per le differente concezione della lingua latina di Varrone e Dionigi, cf. dettagliatamente BRIQUEL, l.c. (n. 3), p. 1037-1038.

16 Pascucci, l.c. (n. 3), p. 343-344; Collart, o.c. (n. 3), p. 240-250. 
dell'Italia così come è delineata da Varrone e la sua teorizzazione linguistica sulle origini del Latino. Come è noto, Varrone identifica nei Sabini i più antichi abitatori d'Italia, accostandoli, per altri versi, anche agli Enotri ${ }^{17}$ e agli Aborigeni. Quest'ultimi sarebbero anzi, nella visione varroniana così come è riassunta da Dionigi di Alicarnasso (II, 48), progenitori dei Sabini stessi, i quali dunque sarebbero presentati dal Reatino come la popolazione più antica, e dunque autoctona, d'Italia ${ }^{18}$.

Tra i molteplici valori che i Sabini erano in grado di fornire ai Romani compariva dunque quello dell'autoctonia ${ }^{19}$, poiché essi, secondo la testimonianza di Catone $^{20}$, discendevano dall'eponimo Sabus, figlio del dio epicorio Sancus. Al di là dei rapporti di questa versione con quella che faceva dei Sabini i discendenti degli Spartani $^{21}$, ciò che a noi interessa è l'esistenza di una tradizione, confluita anche in Catone e verosimilmente accettata anche da Varrone, che faceva dei Sabini una popolazione autoctona, in opposizione alla tradizione, probabilmente già nota a Timeo, che accreditava l'origine spartana dei Sabini ${ }^{22}$.

Il dato dell'autoctonia sabina va combinato ${ }^{23}$ con il mitico stemma genealogico, il cui nucleo essenziale doveva circolare già alla fine del IV secolo a. C., che legava i Romani ad altre popolazioni italiche, quali ad esempio i Sanniti, in virtù della

17 SERVIO, ad Aeneidem, I, 532: Oenotria dicta est, ut Varro dicit, ab Oenotro rege Sabinorum. Si è letto in questa testimonianza il tentativo da parte di Varrone di identificare i più antichi abitatori dell'Italia, nella tradizione greca gli Enotri, con i Sabini. CollaRT, o.c. (n. 3), p. 229 e PASCUCCI, l.c. (n. 3), p. 351-352.

18 Dionigi, molto probabilmente a differenza di Varrone, ritiene gli Aborigeni di origine greca, evidentemente mosso dalla necessità di "grecizzare" quanto possibile la genesi di Roma. Musti sottolinea come, verosimilmente, Varrone accreditasse la tesi dell'autoctonia, la quale ovviamente non esclude rapporti posteriori tra Sabini e Greci. D. Musti, "I due volti della Sabina: sulla rappresentazione dei Sabini in Varrone, Dionigi, Strabone, Plutarco", DdA 3 (1985), p. 77-86 (= in Preistoria, storia e civiltà dei Sabini, Rieti, 1985, p. 75-98; D. MusTI, Strabone e la Magna Grecia, Padova, 1994, p. 235-257).

19 Esplicita in questo senso la testimonianza di Strabone V, 3, 1. Sull'autoctonia dei Sabini, cf. in sintesi J. Poucet, Les origines mythiques des Sabins, in Études étrusco-italiques, Louvain, 1963, p. 155-225, in part. p. 213-215.

20 Dionigi di Alicarnasso, II, 49, 2 = CAtone, Origines, fr. 50 P.

$21 \mathrm{Su}$ tutto questa vedi esaustivamente C. LETTA, "L'Italia dei mores romani nelle Origines di Catone", Athenaeum 62 (1984), p. 3-29, 416-439, in part. p. 437, secondo cui quella di Catone sarebbe la risposta "autoctonista" a chi sosteneva le origini greche, e più in particolare spartane, dei Sabini.

22 Su cui si veda LETTA, l.c. (n. 19), p. $432-438$ e recentemente, C. LETTA, "I legami tra i popoli italici nelle Origines di Catone", in G. UrSo, M. SORDI (éds), Patria diversis gentibus una? Unità politica ed identità culturale nell'Italia antica, Atti del Convegno, Cividale del Friuli 2007, Pisa, 2008, p. 171-195, in part. p. 173.

23 Cf. F. Russo, Roma mater Italiae ac parens urbs, c.d.s., in cui si affronta il problema della diffusione del concetto di Italia nel corso del III secolo a. C., soprattutto come definizione del dominio spettante a Roma nelle relazioni romano-cartaginesi e tra i Romani e Pirro. 
comune discendenza dai Sabini ${ }^{24}$. Grazie al concetto della syngeneia, l'elemento sabino costituiva la chiave di volta di questa complessa struttura etnica, accomunando le diverse popolazioni italiche di questa "famiglia" ancor prima della diffusione del concetto di Italia intesa come patria comune.

Come è stato ben mostrato da Mazzarino ${ }^{25}$, l'Italia a cui ci si riferì negli anni della campagna annibalica va identificata non con l'Italia "geograficamente" intesa, cioè con l'Italia dalle Alpi fino allo stretto di Messina ${ }^{26}$, bensì con "l'Italia propriamente detta" menzionata Appiano ${ }^{27}$ (Hann., 8, 34), con l'Italia ad ovest dell'Appennino (esclusa l'Etruria) ${ }^{28}$.

Il fatto che in questa "vera Italia" si localizzino quelle popolazioni che derivano dai Sabini determina una sovrapposizione tra il concetto d'Italia e quell'insieme di popolazioni italiche che, anche se forse in momenti diversi della tradizione, trovarono nei Sabini la loro origine più antica.

Questa Italia coincide con l'Italia "morale" delineata da Catone ${ }^{29}$, da cui sono esclusi i Liguri, i Galli egli Etruschi ${ }^{30}$; ed è proprio a partire dall'impostazione ideologica delle Origines catoniane che è possibile forse dire qualcosa di più preciso sul significato delle parole di Varrone nella testimonianza di Giovanni Lido.

Catone, che molto verosimilmente conosceva non solo la discendenza dei Sanniti dai Sabini ma anche quella dei Marsi dai Sabini ${ }^{31}$, così come la derivazione sabina per altri popoli appenninici, valorizzava in particolare il contributo dato dai

24 Si veda F. Russo, Pitagorismo e Spartanità. Elementi politico-culturali tra Roma, Taranto e i Sanniti alla fine del IV secolo a.C., Campobasso, 2007, p. 36s. Ivi indicazioni bibliografiche.

25 S. MAZZARINo, Il pensiero storico classico, vol. 2, Bari, 1966, p. 212s. Si veda anche G. BRIZZI, "Appennino e Termopili”, in R. ChEvAlliER (éd.), Littérature gréco-romaine et géographie historique. Mélanges offerts à R. Dion, Paris, 1974, p. 315-324 e da ultimo W. V. HARRIS, "Quando e come l'Italia divenne per la prima volta Italia? Un saggio sulla politica dell'identità", SS 48 (2007), p. 301-322.

26 Cf. Catone, Fr. 85 P e Polibio, III, 54, 2. Sulla tensione tra Italia in senso geografico e Italia in senso morale, e più in particolare sulla non coincidenza delle due accezioni, cf. E. GABBA, "Il problema dell'unità dell'Italia romana", in La Cultura italica, Atti del Convegno della S. I. G., Pisa, 1977, 1978, p. 11-27; LETTA, l.c. (n. 19), p. 418.

27 Più dettagliatamente e con rimandi bibliografici, LETTA, l.c. (n. 19), p. 418.

28 Mazzarino collega la testimonianza di Appiano al rituale della sepoltura di una coppia di Greci e una coppia di Galli menzionato da Livio (XXII, 57, 6). MAZZARINO, o.c. (n. 23), p. 213.

29 Sul ruolo degli Italici nell'ideologia catoniana, cf. F. WULfF AlONSO, "Notas sobre el mundo italico en la ideologia romana: Lucilio 1088m y Caton el Censor", Baetica 7 (1984), p. 211-218, in part. p. 216.

30 LetTA, L'Italia, p. 416-417. Visto il giudizio che Catone aveva degli Opici, forse anche quest'ultimi saranno da aggiungere alla lista delle popolazioni escluse dall'accezione morale di Italia (chiaramente non da quella geografica). Per gli Opici in Catone, cf. F. Russo, M. BARBERA, "Da 'Otıxò a Oscus: osmosi semantica ed evoluzione lessicale", SSL 42-43 (2004-2005), p. 89-120.

31 LeTtA, l.c. (n. 19), p. 422-423. 
Sabini ai Romani ${ }^{32}$. Il rapporto che legava i Romani ai Sabini era sì di carattere etnico, prima di tutto a causa dell'episodio del ratto delle Sabine, ma aveva anche e forse soprattutto, nella visione di Catone, una valenza morale, poiché i Sabini avevano

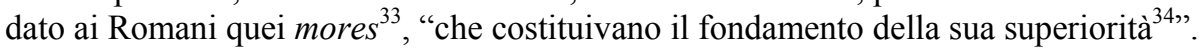

Mentre il legame di tipo "etnico", nel senso di comune discendenza di stirpe, fu quello a cui fece riferimento, ad esempio, Ponzio Sannita ${ }^{35}$ (i Romani sono syngeneis dei Sanniti poiché entrambi i popoli derivano dai Sabini), da parte romana, poiché si evitò sistematicamente di parlare in termini di syngeneia con le altre popolazioni italiche ${ }^{36}$, si preferì, a mio avviso, sottolineare ed evidenziare l'apporto morale dei Sabini ai Romani, apporto che, come testimonia Catone nelle sue Origines (fr. 76 P), fu condiviso da tutta l'Italia, quell'Italia intesa dal Censore in senso morale. Aggiungiamo che tra le virtù trasmesse dai Sabini ai Romani doveva comparire anche il forte legame tra i Sabini e l'Italia, espresso dal valore dell'autoctonia.

In particolare, fu la propaganda anti - annibalica a fare perno più esplicitamente sul concetto di unità etnico - culturale del fronte romano - italico il cui l'orizzonte di riferimento fu quello specificamente italico-sabino e la cui cifra essenziale fu rappresentata dall'autoctonia e dalla comunanza di linguaggio e altri costumi, in contrasto rispetto al comune nemico cartaginese.

Tale visione ebbe anche una sua espressione linguistica: fra i vari motivi per cui l'esercito di Annibale fu stigmatizzato ricorreva anche quello della mancanza di unità culturale. Tracce di questa polemica ${ }^{37}$ si ravvisano nei carmina Marciana ${ }^{38}$ (come testimonia l'uso del concetto di alienigena) ed in un discorso che Gaio Terenzio Varrone rivolse ai Capuani dopo la sconfitta di Canne ${ }^{39}$ : Poenus hostis, ne Africae quidem indigena, ab ultimis terrarum oris, freto Oceani Herculisque columnis, expertem omnis iuris et condicionis et linguae prope humanae militem trahit. Annibale (perché è a lui e al suo esercito che si riferiscono le parole di G. Terenzio Varrone, e non a Cartagine in sé) non ha patria, è straniero rispetto ai Romani ma anche rispetto all'Africa, il suo esercito viene da lontano ${ }^{40}$ (e qui c'è il

32 LETTA, l.c. (n. 19), p. 430 e n. 235.

33 Fr. 51 P: Sabinorum ...mores populum Romanum secutum... Cato dicit.

34 LETTA, l.c. (n. 19), p. 430.

35 Appiano, Samnitikà, 4, 5. Sul problema della syngeneia italico-romana cf. Russo, o.c. (n. 21) e F. RUSSO, "Il motivo della consanguinitas tra Romani ed Italici nella propaganda graccana”, AR c.d.s.

36 RUSSO, o.c. (n. 21).

37 G. URSO, "Il concetto di alienigena nella guerra annibalica", in Emigrazione e immigrazione nel mondo antico, CISA 20, Milano, 1994, p 223-236; G. URSO, "Pro Italia vobis est pugnandum: Annibale al Ticino", RSA 33 (2003), p. 67-91. Più recentemente, F. RuSsO, "I carmina Marciana e le tradizioni sui Marcii”, PP 60 (2005), p. 7-35.

38 Russo, l.c. (n. 34).

39 LIVIO, XXIII, 5, 11.

40 Si veda il discorso di Marco Minucio (LIVIO, XXII, 14, 4-5), in cui si dice: Poenus advena, ab extremis orbis terrarum terminis...progressus. L'idea che l'esercito di Cartagine raccogliesse soldati provenienti da molto lontano e un po' da ovunque si ritrova già nella propaganda di Timoleonte da Corinto, secondo cui l'esercito di Cartagine proviene 
richiamo al vomica quae gentium venit longe del secondo dei carmina marciana), e soprattutto è composto da persone di ogni estrazione ${ }^{41}$. Al contrario, il fronte italico possiede tutte quelle caratteristiche negate ad Annibale, tra cui in particolare l'autoctonia ${ }^{42}$. La mancanza di quest'ultima caratteristica rende ingiusti, agli occhi dei Romani, i tentativi di Annibale di ridurre l'Italia a provinciae Africae ${ }^{43}$.

Ad Annibale dunque viene anche negato il diritto di "autoctonia" che, invece come si è visto altrove, aveva un notevole peso ideologico nelle pretese di Roma sull'Italia, soprattutto nel contesto delle guerre puniche.

Né d'altra parte ci troviamo di fronte ad un'invenzione romana: il tema dell'autoctonia ebbe particolare fortuna nella propaganda greca ${ }^{44}$, poiché essa, tra $\mathrm{i}$ vari significati che poteva assumere, era funzionale anche alla rivendicazione, da parte dei Greci, di un'identità etnica ${ }^{45}$ e culturale che a sua volta giustificava e sosteneva i diritti ancestrali di chi si riteneva autoctono a difendere e conservare il dominio sul proprio territorio (sentito appunto come terra d'origine) contro usurpatori stranieri, esterni $^{46}$. Il rivendicare l'autoctonia di un popolo dunque costituiva, anche nell'ottica romana, un'affermazione ideologicamente forte, che non esauriva certo il suo pieno

"dall'Oceano e dalle Colonne d'Ercole" (Plutarco, Timoleonte, 20, 8). Sicuramente a Roma in età annibalica era nota questa notizia, forse di tradizione timaica. Cf. A. Momigliano, "Linee per una valutazione di Fabio Pittore", in Terzo contributo alla storia degli studi classici e del mondo antico, vol. 1, Roma, 1966, p. 55-68, in part. p. 61-62 (già in RAL 15 (1960), ser. 8, p. 310-320).

${ }^{41}$ Che questa fosse una caratterizzazione particolarmente nota del fronte annibalico è confermato dalla testimonianza di Polibio, Polibio elogia Annibale, perché in sedici anni di guerra in Italia, non gli si è mai ribellato contro l'esercito, pure così composito (XI, 19, 3-4).

42 Così anche M. SORDI, "Terra Etruria e Terra Italia", in C. BEARzot, F. LANDUCCI, G. ZECCHINI (éds), Gli stati territoriali nel mondo antico, Milano, 2003, p. 127-134, in part. p. 131-132.

43 LiVIO, XXIV, 47, 6.

44 In questo senso è emblematico Aristotele, Rhet., I. 5 (BEKKeR 1360b). Vedi anche Diodoro, I, 9, 3; E. J. Bickerman, "Origines gentium”, CPh 47 (1952), p. 65-81 (in part. p. 76-77); N. LORAUX, "L'autochthonie: une topique athénienne: le mythe dans l'espace civique", AESC 34 (1979), p. 3-26; V. Rosivach, "Autochthony and the Athenians", CQ 37 (1987), p. 294-306; E. GABBA, Dionysios and the History of Archaic Rome, Berkeley/Los Angeles/Oxford, 1991, in part. p. 104-106; N. LORAuX, Né de la terre. Politique et autochthonie à Athènes, Paris, 1996; M. SORDI, "Propaganda e confronto politico", in A. D'Atena, E. Lanzillotta (éds), Alle radici della democrazia: dalla polis al dibattito costituzionale contemporaneo, Roma, 1998, p. 57-67; D. BRIQUEL, "Les Romains ne sont pas des autochthones", in B. Chevallier, R. Poignault (éds), Présence de Tite-Live. Hommage au Professeur P. Jal, Tours, 1994, p. 67-78.

45 Sulle numerose testimonianze letterarie della fortuna di questo tema, cf. la sintesi di Loraux, o.c. (n. 42), p. 27-48.

46 Fondamentale l'ampia analisi di E. MONTANARI, Il mito dell'autoctonia. Linee di una dinamica mitico-politica ateniese, Roma, 1981. Più recentemente, C. BEARZOT, "Autoctonia, rifiuto della mescolanza, civilizzazione da Isocrate a Megastene", in T. GNOLI, F. Muccioli (éds), Incontri tra culture dell'Oriente ellenistico e romano, Atti del Convegno, Ravenna, 2005, Milano, 2007, p. 7-21. 
significato e portato propagandistico nelle ricostruzioni mitiche delle origini della città. Nel periodo annibalico, la rivendicazione dell'autoctonia del fronte italico, grazie alla comune discendenza sabina, forniva ai Romani un'arma propagandistica, di origine greca, contro le pretese di Annibale e ancor di più contro pericolose alleanza tra quest'ultimo e le comunità italiche. E' infatti chiaro che Roma dovette produrre o perlomeno appoggiare una propaganda in grado di cementare $\mathrm{i}$ suoi rapporti con le comunità alleate, il cui passaggio al fronte annibalico poteva risultare esiziale per Roma stessa.

Il concetto di autoctonia e quello di lingua comune sono due caratteristiche strettamente connesse e quasi consequenziali, connaturate al Sabino ${ }^{47}$, in linea con la visione della comune origine sabina di alcune popolazioni italiche.

3. Come si è detto, è evidente lo sforzo di Varrone di porre i Sabini al livello più antico e originario, e quindi autoctono, della storia d'Italia: gli Aborigeni, muovendo dal reatino, cacciarono dai colli laziali i Siculi con l'aiuto dei Pelasgi, concessero il Palatino all'arcade Evandro e si fusero poi con i Troiani di Enea ${ }^{48}$. Non torneremo sulla complessa questione della "preistoria" di Roma nelle pagine varroniane; qui importa soprattutto notare che il dato dell'autoctonia, intrinseco all'immagine dei Sabini, doveva avere anche una sua validità sul piano linguistico: la lingua dei Sabini doveva dunque apparire come autoctona e assai antica, grazie al fatto stesso che essi erano (fra) i più antichi abitatori di Italia ${ }^{49}$.

Questo particolare aspetto della lingua sabina, finora non valorizzato dalla critica moderna, può fornire una nuova chiave di lettura per il problematico passo di Giovanni Lido.

Nel frammento in questione, Varrone non sta semplicemente discutendo la sua tesi delle origini del Latino, quanto piuttosto l'apporto di lingue "straniere", cioè non autoctone rispetto all'Italia, al Latino ${ }^{50}$, o per meglio dire alla sua base autoctona, sia essa da identificare o meno con la lingua dei Sabini. In accordo ad una posizione della teoria linguistica antica di origine greca ${ }^{51}$, che riconosceva anche nel Greco la presenza di apporti "barbari”, anche per quanto riguarda il Latino ritengo che Varrone

47 Ricordiamo che Varrone, come si è visto, fa dell'Osco un derivato del Sabino.

48 Cf. il fondamentale lavoro di F. Della CoRTE, "L'idea della preistoria in Varrone", in Atti del Congresso Internazionale di Studi Varroniani, Rieti 1974, 1976, vol. 1, p. 111-136, che ha raccolto tutte le testimonianze varroniane relative alla più antica storia di Roma e dei Sabini.

49 Secondo PASCUCCI, l.c. (n. 3), p. 345, Varrone "identificò coi Sabini gli irradiatori della più antica civiltà, sorta primariamente nella regione di Rieti ad opera degli Aborigeni".

50 In questa direzione ci spinge anche il senso generale della testimonianza di Giovanni Lido, al cui interno si trova la citazione varroniana: l'autore infatti, descrivendo la caratteristica uniforme del praefectus praetorio enumera i nomi latini delle singole parti della cintura, ma, dovendo citare il nome "esotico" usato dai Romani per indicare l'intera uniforme, menziona la teoria di Varrone relativa all'apporto di Etrusco, Greco (Eolico) e Gallico al Latino. Si parla dunque di termini "stranieri" adottati comunemente dal Latino, ed è in questa discussione che deve essere compreso il rimando varroniano.

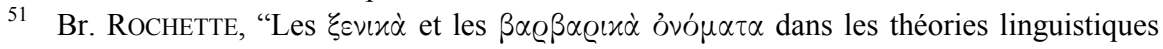
gréco-latines", $A C 65$ (1996), p. 91-105. 
proponesse uno schema simile, al cui interno il Greco, l'Etrusco e il Gallico costituivano il côté "straniero" della formazione latina.

A proposito della lingua parlata da Romolo, Collart si esprime così: "à l'origine, Romulus et ses compagnons parlent entre eux ce qu'on pourrait appeler le 'prélatin'. Sur la nature de ce latin primitif, Varron ne nous donne, il est vrai, aucun renseignement. D'autre part, Romulus et les siens n'ignorent pas non plus la langue qu'Evandre et son entourage avaient diffusée entre les barbares. Il s'ensuit alors pour les Romains un langage hybride, teinté de grec, doublement hybride même, puisque bientôt des rapports amicaux avec les habitants de la Sabine permettent aux AlbanoLatins de prendre contact avec les mœurs et le vocabulaire de leurs voisins ${ }^{52}$. Secondo lo studioso, si può ritenere, sulla base di altre testimonianze varroniane, che in questa primissima fase i Sabini abbiano avuto una notevole importanza nella formazione della lingua latina (il Latino "profuma" di Sabino ${ }^{53}$ ), non solo perché essi fornirono ai Romani buona parte del loro vocabolario (soprattutto per alcuni settori, come ad esempio quello cultuale), ma anche perché grazie a loro i Romani adottarono elementi lessicali di origine etrusca e greca ${ }^{54}$. Come si vede, il Sabino è posto da Varrone su un piano cronologico più antico, contemporaneo al "prelatino" $\mathrm{e}$ soprattutto antecedente al Greco e all'Etrusco. Esattamente in coincidenza con la ricostruzione varroniana delle origini di Roma.

Il Greco e l'Etrusco (e a maggior ragione il Gallico) devono essere considerate lingue "straniere", soprattutto se si assume come punto di vista quello dell'Italia sabina, cioè centro appenninica: in altre parole il concetto di Italia alla base dell'idea di "vera Italia", così come indicato sopra. Il Greco, l'Etrusco e il Gallico hanno qualcosa in comune che non potrebbe mai essere condiviso dal Sabino, e cioè non sono lingue "propriamente" italiche nella visione varroniana, e quindi non possono esprimere il concetto di autoctonia.

L'interpretazione qui proposta del passo varroniano richiama significativamente alcune posizioni ideologiche assunte da Roma nei confronti degli altri popoli italici nel corso del III secolo, quando, come si è visto in maniera approfondita altrove, si accentuò l'idea di un'identità italica di base appenninica, da cui erano esclusi Greci, Galli ed Etruschi, così come è icasticamente rappresentato dal noto episodio della sepoltura rituale di una coppia di Galli e una di Greci ${ }^{55}$, il cui significato, come è stato mostrato, si esprime proprio in relazione alla definizione di un'alterità etnica rispetto al concetto di Italia ${ }^{56}$.

52 Così Collart, o.c. (n. 3), p. 249, "le latin, résultante de grec, de latin et de sabin, se charge aussi d'éléments étrusques soit directement, soit plutôt encore par l'intermédiaire des Sabins". Concordo con il Collart, ma non per quanto riguarda l'equiparazione tra Greco e Sabino, essendo il primo una lingua straniera, a differenza del secondo, autoctono e originario. Per l'intermediazione del Sabino tra Greco e Latino, cf. anche PASCUCCI, l.c. (n. 3), p. 340-344.

53 Cf. De lingua Latina, V, 9, 17. Collart, o.c. (n. 3), p. 249.

54 FUnAIOLI, o.c. (n. 1), p. 311 fr. 295.

55 Livio, XXII, 57, 4-6.

56 Vd. in particolare A. FrASChETTI, "Le sepolture rituali nel Foro Boario", in Le délit religieux dans la cité antique, Roma, 1981, p. 51-115. 
Così come i Sabini costituivano il coagulante etnico di un fronte italico esteso che comprendeva anche i Romani, fornendo a questi la patente di autoctonia rispetto all'Italia, allo stesso modo la loro lingua, proprio perché autoctona, non poteva essere messa sullo stesso piano di quella greca, etrusca o gallica ${ }^{57}$ : mentre il sabino costituiva il coté locale ed autoctono del latino, le altre tre ne rappresentavano per così dire il lato esotico, così come Greci, Etruschi e Galli erano stranieri rispetto all'Italia stessa.

Ciò non significa che Varrone identificasse il Sabino con la "lingua vernacolare"; in questo senso, le indicazioni che possediamo sono troppo esigue per pronunciarci o meno in favore di questa ipotesi. Semmai, è importante ribadire che il Sabino, come parte costituente del Latino, possedeva una qualità, l'autoctonia e cioè l'essere una lingua propriamente italica, che in nessun modo poteva essere condivisa dal Greco, dall'Etrusco o dal Gallico ${ }^{58}$.

Varrone, nel frammento di Giovanni Lido, menzionerebbe proprio l'apporto esterno, cioè non autoctono, al Latino: per questa ragione la menzione del Sabino (o dell'Osco come riferimento indiretto al Sabino) sarebbe stata inopportuna, mentre la sua assenza risulta del tutto comprensibile, soprattutto alla luce della teorie linguistiche ed etniche varroniane.

Gli stretti collegamenti tra la teoria varroniana ed il concetto di Italia così come si determinò nella seconda metà del III secolo, e più in particolare in età annibalica, suggeriscono che l'impianto ideologico sotteso alla ricostruzione linguisticoetimologica varroniana non sia un apporto originale del Reatino, quanto piuttosto un ricordo preciso di una visione globale delle popolazioni italiche, ivi compresi i Romani, già circolante nel III secolo a. C., al cui interno i Sabini occupavano un ruolo centrale ed essenziale $^{59}$. In altre parole, la teoria linguistica varroniana riposerebbe su

57 Come detto, Varrone sosteneva il ruolo primario dalla lingua sabina nella formazione del Latino, anche perché il Greco, come l'Etrusco, fu noto ai primi Romani grazie all'intermediazione sabina (Res rustica, III, 1).

58 Ha ragione senza dubbio BRIQUel, l.c. (n. 3), p. 1039, a mettere in guardia da una visione eccessivamente pansabinista della lingua latina, poiché mai Varrone afferma che il Latino sia da identificare col Sabino. D'altra parte, il Reatino non manca di sottolineare l'apporto, anche lessicale, della lingua dei Sabini a quella dei Latini. Per quanto riguarda l'interpretazione prospettata dallo studioso, secondo cui la duplice e apparentemente pleonastica menzione degli Etruschi / Tusci celerebbe in realtà un riferimento alla distinzione tra termini di origine etrusca più recente e termini di origine più antica, vediamo bene che la ricostruzione qui proposta collimerebbe agevolmente con quanto ipotizzato da Briquel, poiché in ogni caso si ribadirebbe il carattere "straniero" della lingua etrusca. Si noti però che non si può escludere, più semplicemente, che Giovanni Lido, riassumendo il testo varroniano, abbia frainteso il senso originario, poiché è certo che Varrone faceva dei Tusci gli abitanti dell'Etruria (De lingua latina, V, 2, 2). Una qualche incertezza del testo originario (ad esempio l'uso dell'etnonimo Tusci in connessione con il toponimo Etruria) potrebbe aver ingenerato una certa confusione, non essendo trasparente, dal punto di vista lessicale, il legame tra etnonimo e toponimo. In ogni caso, rimane stabile il riferimento alla lingua degli Etruschi.

59 Questa visione, non più attuale dopo la fine della guerra annibalica, fu recuperata da Catone, che propose un quadro più sistematico, coerente e soprattutto ideologicamente conno- 
una concezione etnica codificata in forma embrionale alla fine del IV secolo e ampiamente circolante nel corso del III secolo. L'aspetto linguistico sarebbe stato parte integrante di questa concezione, poiché la comune discendenza etnica avrebbe determinato come conseguenza logica la condivisione di tratti linguistici comuni, lingua compresa.

Universität Konstanz

Federico RUSSO

Fachgruppe Geschichte

D-78457 Konstanz

Federico.Russo@uni-konstanz.de 